\title{
Valveless interface for two-dimensional reversed-phase microcromatography ( $\mu$-RPLC)-capillary zone electrophoresis (CZE)
}

\author{
O. Cabaleiro, M. Lores and R. Cela* \\ Dpto. Química Analítica, Nutrición y Bromatología, Facultad de Química, Universidad de Santiago de \\ Compostela, Avda. Das Ciencias s/n, 15706-Santiago de Compostela, Spain
}

\begin{abstract}
Single stage separation systems have limitations in resolving multicomponent mixtures and two-dimensional systems have higher peak capacities. A valveless interface for hyphenating Reversed-phase Microchromatography ( $\mu$-RPLC) and Capillary Zone Electrophoresis (CZE) -highly orthogonal separation methods- is presented, which main innovation is a third electrode. Design and operation of the interface as well as some preliminary results obtained by combining FIA and $\mu$-RPLC with CZE are shown.
\end{abstract}

Key words. Two dimensional systems - micro-LC - CZE - valveless interface.

\section{Introduction}

Since the first studies on two-dimensional (2D) separation systems published by Bushey and Jorgenson [1] further research on this subject has been done [2-4].

Mathematical models [5-7] predicted that as sample complexity increases, high efficiency separations are necessary for its complete resolution. Two-dimensional separations have peak capacities approximately equal to the product of the capacities for each dimension [7].

To couple two techniques successfully, they should be orthogonal [1] (their separations mechanisms should be based on different properties), making RP-HPLC and CZE good candidates for being connected. The dissimilarity of these two techniques increases the difficulty of coupling, while enhances the power of this particular 2-D system.

Lemmo and Jorgenson designed a transverse flow gating interface for coupling microcolumn liquid chromatography $(\mu-$ RPLC) and capillary zone electrophoresis (CZE) $[2,3]$ in a "comprehensive way" (all of the effluent from the first dimension is reanalysed in the second one). The relative advantages of using microcolumns rather than conventional columns are well known $[9,10]$. Subsequently, Hooker and Jorgenson [4] modified that interface by changing the building material for a transparent one, which allows direct observation and routine manipulation of the system connections.

We propose a flow gating interface based on this last design, but with significant modifications. The main innovation is the addition of a third electrode: the system makes use of a switch allowing the swap between two electrodes, so the system can run like two independent dimensions. The full sample volume does not go through the second dimension, the switch allows to transfer several discontinue fractions of LC effluent to the CZE capillary obtaining a more diverse sampling of the first dimension.

\section{Experimental}

STANDARDS: 4-hydroxibenzoic and 3,4,5 trimethoxycinnamic were of $97 \%$ purity from Fluka (Buchs, Switzerland). All solutions were prepared in water (Milli-Q, Millipore, Milford, MA, USA).

BUFFERS: CZE buffer (buffer A) was $20 \mathrm{mM}$ $\mathrm{Na}_{2} \mathrm{~B}_{4} \mathrm{O}_{7} \cdot 10 \mathrm{H}_{2} \mathrm{O}$ adjusted to $\mathrm{pH} 9.2$ with $\mathrm{NaOH}$. Buffer $\mathbf{B}$ (used as mobile phase in isocratic elution) was $10 \mathrm{mM}$ $\mathrm{NaCH}_{3} \mathrm{COO} .3 \mathrm{H}_{2} \mathrm{O}$ adjusted to $\mathrm{pH} 2.0$ or 4.0 with $\mathrm{HCl}$. Buffer solutions were made with Milli-Q water and filtered through $0.22 \mu \mathrm{m}$ membrane filters from Millipore. The solid reagents were obtained from Merck (Darmstad, Germany).

Experimental set-up is depicted in figure 1.

\section{Interface design}

Figure 2 shows a flow gating interface scheme detailing its dimensions. The interface is home-built up from polished methacrylate; the transparent material allows a simpler capillaries handling, which is an important advantage because

* Correspondence and reprints.

Received February 26, 1999; revised June 17, 1999; accepted June 18, 1999. 


\section{Original articles}

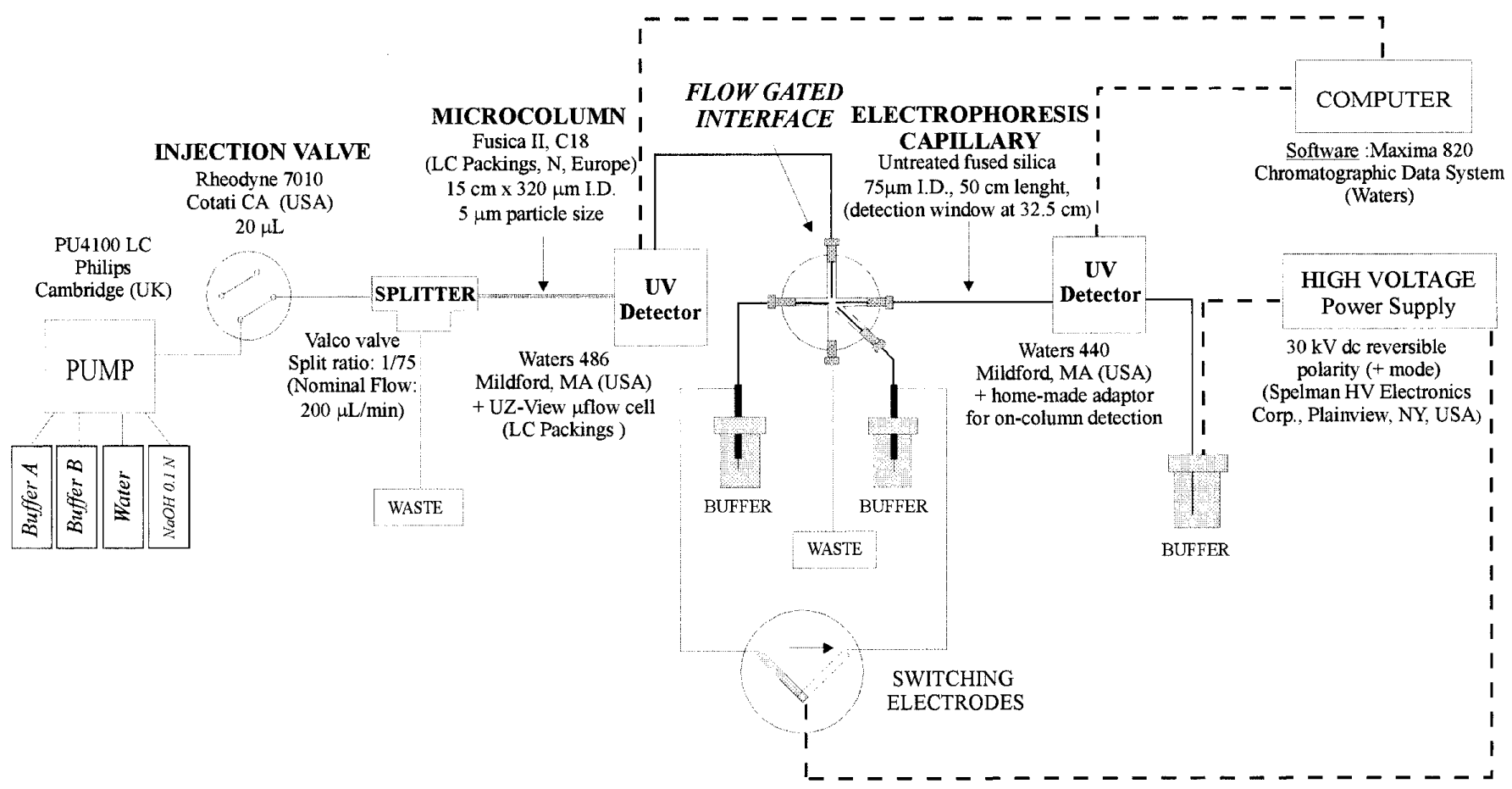

Fig. 1. Instrumental set-up for 2D Micro-RPLC-CZE with all experimental details.

the gap between them is critical for successfully transfer the sample to CZE.

The system consists of three electrodes: one of them is held at electric ground (cathode for the CZE system), the other two (anodes) are connected to a high voltage power supply and inserted into vials containing buffer A; a switch allows the swap between them. At "inject position" (Fig. 2a), an electrokinetic injection to carry the sample from the $\mu$-HPLC effluent to the second dimension is performed by applying a constant voltage to one electrode for an instant, enough time for the sample to reach the interface; then making a switch to the other electrode to "run position" (Fig. 2b), the system runs like a simple electrophoresis system preventing electromigration from $\mu$-HPLC to CZE capillary. After the injection is performed, the transverse flow from HPLC pump is carried to waste. The first dimension can run independently of the CZE system (position 2a) and vice versa (position $2 b$ ).

\section{System pre-conditioning}

$\mathrm{NaOH}(0,1 \mathrm{~N})$ for conditioning the capillary walls followed by fresh buffer are pumped through the CZE capillary daily. Before the first 2D run, the sample is injected in the CZE capillary to check the electrophoretic analysis and injection performance. The CZE capillary is pumped again with fresh buffer before connecting up the 2D system.
The microcolumn must be stabilised before being connected to the capillary and the chromatographic retention time is determined by a previous operation of the $\mu$-HPLC system independently.

\section{Results and discussion}

\section{FIA mode}

To set-up the FIA system an empty capillary ( $75 \mu \mathrm{m}$ ID) is placed instead of the microcolumn. Both, FIA and electrophoresis capillaries have been filled with $100 \%$ buffer A, pumped from the HPLC pump reservoirs.

Sample introduction was performed as it was described above (Interface Design Section). CZE injections (10 kV) gave $30 \mu \mathrm{A}$ current, but in RUN position the current was 60 $\mu \mathrm{A}$ for the same voltage. The different length of capillaries $\mathrm{A}$ and $\mathrm{B}$ (Fig. 2a and 2b) justifies this increment. The single dimension electropherogram (Fig. 3) shows the separation of the two compounds that come out overlapped from the FIA capillary, displaying several consecutive and reproducible injections.

Tetraborate is allowed in FIA analysis but it will degrade the $\mathrm{C}_{18}$ stationary phase. Water in the first dimension produces the same results, but the system is unstable, allowing only two to three injections of the sample during the same 


\section{Original articles}

\section{INJECT POSITION (2a)}

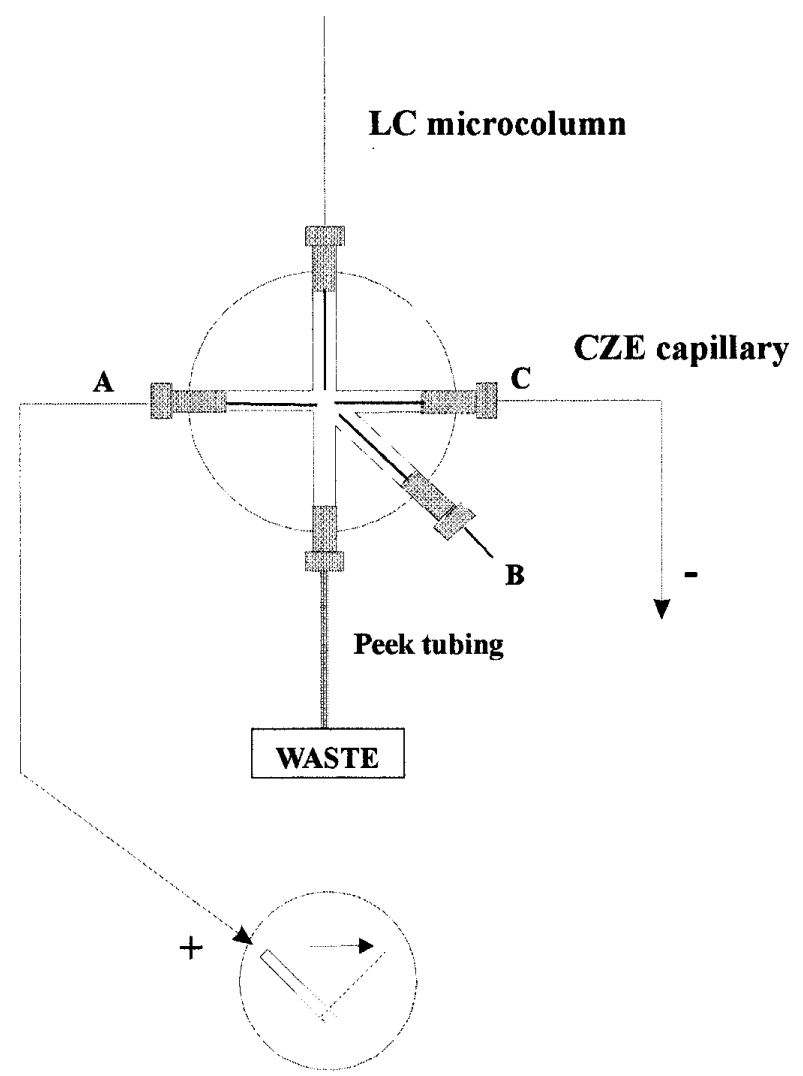

RUN POSITION (2b)

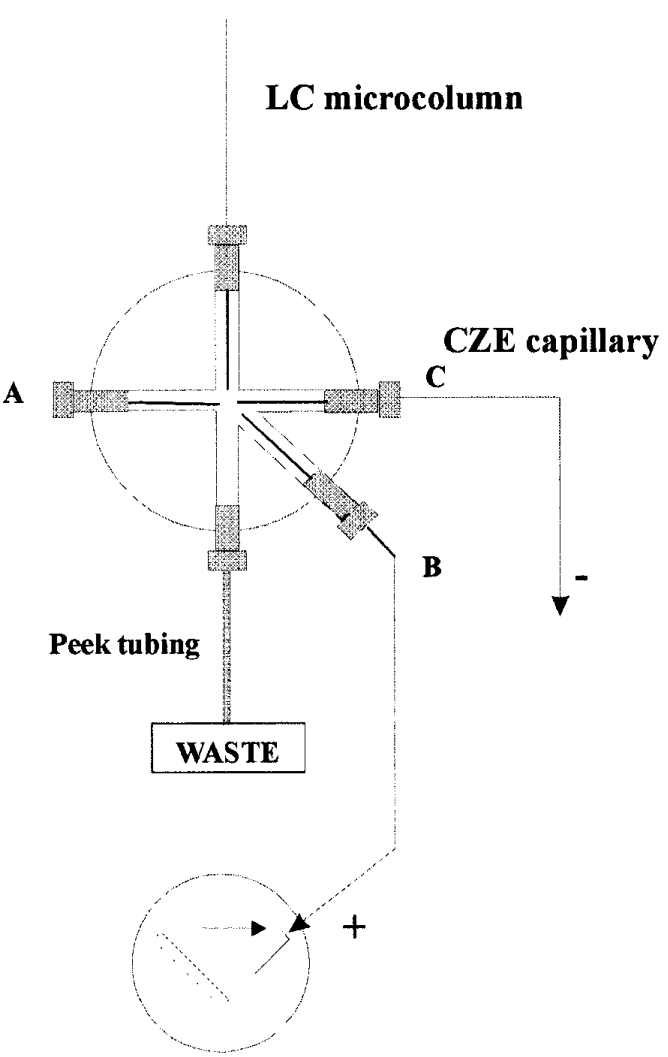

Fig. 2. Valveless interface scheme: disk $(25.4 \mathrm{~mm}$ diameter and $12.7 \mathrm{~mm}$ thick, with five $1.59 \mathrm{~mm}$ channels; Teflon connectors $(0.18 \mathrm{~mm}$ ID, $1.59 \mathrm{~mm}$ OD). a) At INJECT position, the voltage is applied between electrodes A and C, allowing analyte eluting from LC column to flow across the CZE capillary and to be electrokinetical injected. b) At RUN position, the voltage is applied between electrodes B and $\mathrm{C}$, the mobile phase from the pump flows through the interface and carries LC effluent to waste. The system runs like a simple CZE.

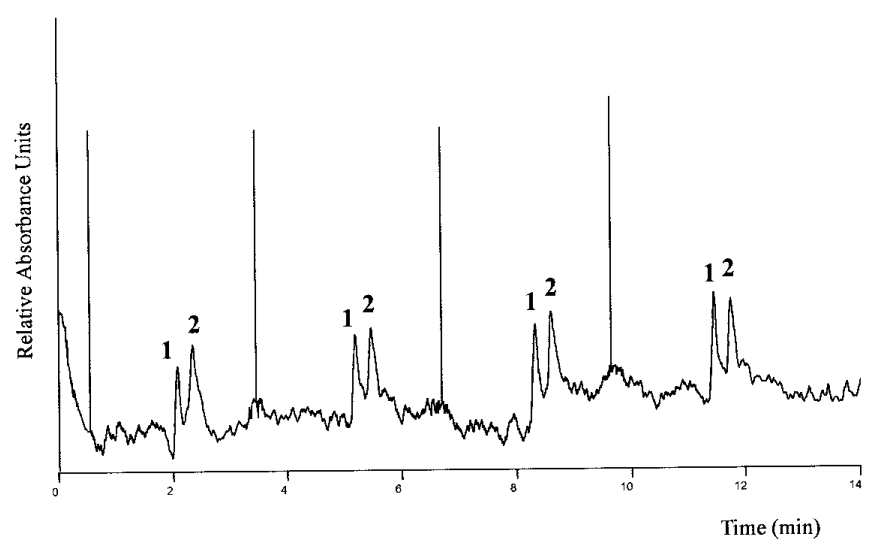

Fig. 3. Electropherogram got by running $100 \%$ buffer $\mathrm{A}$ in both dimensions (data in FIA not shown). The spikes show exactly the swap made for injection. (Key: 1 -4-Hydroxibenzoic acid, 2- 3,4,5Trimethoxycinnamic acid). run and causing unsteady current afterwards. The need of keep using a buffer in the first dimension is clear, so an acidic $\mathrm{pH}$ one was tested.

The CZE capillary is filled with buffer A as in previous assays. The mobile phase was held isocratic with $50 \%$ water$50 \%$ acetate $(\mathrm{pH} 2)$. The mixture of CZE buffer and the carrier buffer prevent, in this case, the two polyphenolic compounds electrophoretic separation. This means that the $\mathrm{pH}$ of the carrier buffer in FIA is determinant, having influence in the second dimension separation process. A new acetate buffer adjusted to $\mathrm{pH} 4$ was prepared, in order to provide the best overall CZE separation for the analysed compounds. An isocratic elution of $50 \%$ water- $50 \%$ acetate was performed producing the separation of the model mixture.

Migration times show a slight variability typical of all 2D-runs due to a gradual increase in the electrosmotic flow rather than a scattered type of variation from injection to injection. 


\section{$\mu-R P L C-C Z E$}

The choice of the mobile phase used in the first dimension is critical. As long as a fraction from the LC effluent is injected on the second dimension some microdilution processes occurs: $\mathrm{pH}$ and/or other properties of the electrophoretical buffer can be modified affecting resolution in CZE. The interface is held at RUN position in order to prevent LC effluent flow in the capillary. Inmediately before the elution of the first peak from the RP column (isocratic, $100 \%$ water), the switch to the INJECT position was done maintaining the voltage at $10 \mathrm{kV}$. This position is held until the unresolved peak pair is completely eluted from the microcolumn; then the rotation to the initial position is done again, working now the system as CZE (100\% tetraborate), independently of the RPLC system.

Water as mobile phase causes CZE current unstability (like in the FIA system) when several injections have been made. Occasionally, the capillary must be pumped with fresh buffer before the next injection, thus limiting on line switching. When the mobile LC phase carries a buffer, current gaps can be avoided. Figure 4 shows the chromatogram and electropherogram of the model mixture (isocratic, $50 \%$ water$50 \%$ acetate buffer $(\mathrm{pH} 4))$ : the two compounds were not separated in the LC dimension (Fig. 4a) but they were baseline resolved in the second one (Fig. 4b). Using this solvent combination, many heartcuttings from the first to the second dimension can be made without significant degradation in the CZE resolving power.

It is important to stand out that the whole system uses only one HPLC pump, there is no need of additional pumps or tees. This is one of the newness of the design presented here: one instrumentation set can work as $\mu$-HPLC (1D), CZE (1D) or both $\mu$-HPLC-CZE (2D) system.

\section{References}

1. Bushey, M. M.; Jorgenson, J. W. Anal. Chem. 1990, 62, 978984.

2. Lemmo, A. V.; Jorgenson, J. W. J.Chromatogr. 1993, 633, 213220.

3. Lemmo, A. V.; Jorgenson, J. W. Anal. Chem. 1993, 65, 15761581.

4. Hooker, T. F.; Jorgenson, J. W. Anal. Chem. 1997, 69, 41344142 . a)

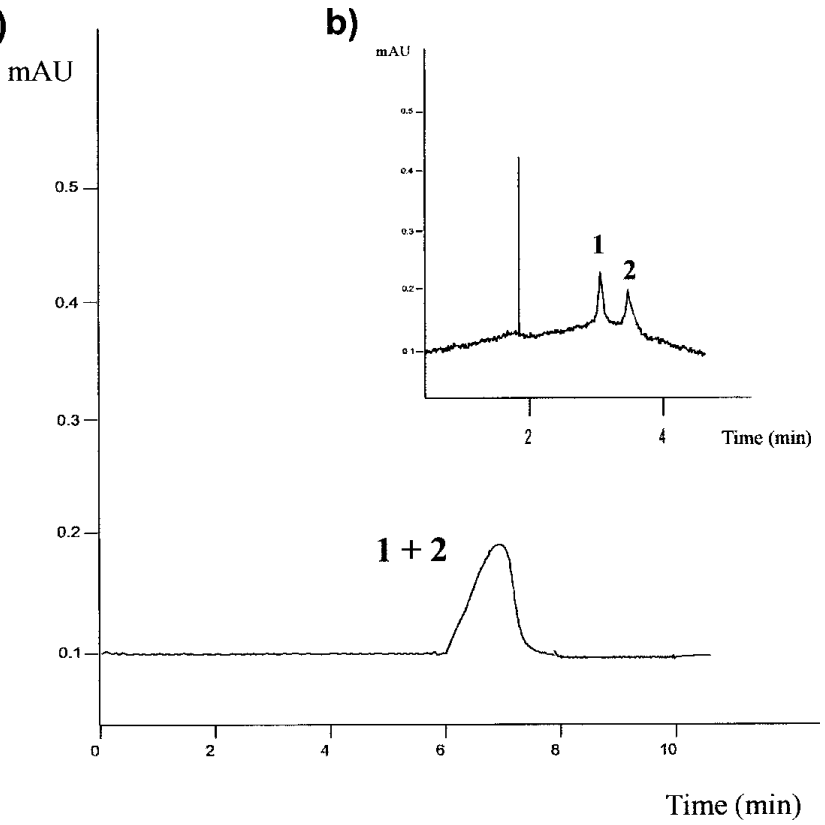

Fig. 4. a) m-RPLC: Chromatogram showing unresolved mixture. Isocratic elution $(50 \%$ water $/ 50 \%$ buffer $\mathrm{B}-\mathrm{pH} 4$ - in the mobile phase). b) CZE: Correspondent electropherogram got with buffer A $(100 \%)$ in the second dimension. Injection and run voltage was 10 kV. (Key: 1 -4-Hydroxibenzoic acid, 2- 3,4,5-Trimethoxycinnamic acid).

5. Giddings, J. C. Anal. Chem. 1984, 56, 1258A-1270A.

6. Davis, J. M.; Giddings, J. C. Anal. Chem. 1985, 57, 21682177.

7. Davis, J. M.; Giddings, J. C. Anal. Chem. 1985, 57, 21782182.

8. Giddings, J. C. HRC CC. J. High Resolut. Chromtogr. Commun. 1987, 10, 319-323.

9. Krejcí, M. "Trace Analysis with Microcolumn liquid chromatography”, 1992, Marcel Dekker, inc. Chromatographic Science Series, Vol. 59.

10. Novotny, M. "Microcolumn High-Performance LiquidChromatography”, 1984, Kucera, P. Ed. J. Chromatogr. Libr., 28 , ch.7. 